\title{
A Three-Dimensional Resonant Triggering Probe for Micro-CMM
}

\author{
Qiangxian Huang ${ }^{1, *}$, Chen Chen ${ }^{1}$, Kui Wu ${ }^{1}$, Liansheng Zhang ${ }^{1}$, Ruijun $\mathrm{Li}^{1}$ \\ and Kuang-Chao Fan 1,2 \\ 1 School of Instrument Science and Opto-Electronic Engineering, Hefei University of Technology, No. 193, \\ Tunxi Road, Hefei 230009, China; jeffchenchen@foxmail.com (C.C.); wukui304@163.com (K.W.); \\ lszhang@hfut.edu.cn (L.Z.); rj-li@hfut.edu.cn (R.L.); fan@ntu.edu.tw (K.-C.F.) \\ 2 Department of Mechanical Engineering, National Taiwan University, No. 1, Sec. 4, Roosevelt Road, \\ Taipei 10617, Taiwan \\ * Correspondence: huangqx@hfut.edu.cn; Tel.: +86-551-6290-5957
}

Academic Editor: Richard Leach

Received: 14 February 2017; Accepted: 12 April 2017; Published: 15 April 2017

\begin{abstract}
To achieve true 3D nano-measurement with sub-nanometer resolution and very low touch force through a micro/nano coordinate measuring machine, a new 3D resonant trigger probe based on a quartz tuning fork is proposed. In this trigger probe, a quartz tuning fork with a microsphere tip vibrates at its resonant frequency, and is used as the sensing element. The resonance parameters of this quartz tuning fork (e.g., vibrating amplitude and resonant frequency) are extremely sensitive to external 3D microforces. The distinguished feature of this probe is its ability to interact with the sample surface in the actual three directions. The microsphere tip of the probe interacts with the sample surface in tapping mode in the $Z$ direction, whereas it interacts in friction mode in the $X$ and $Y$ directions. The dynamic contact mechanism of the probe is based on interfacial force theory, and mechanical models of the interactions between the microsphere tip and sample surface in the $X, Y$, and $Z$ directions are constructed and simulated. The experiment shows that the probe has sub-nanometer resolution in 3D directions and triggers repeatability of approximately $40 \mathrm{~nm}$ in each direction. Theoretical analysis and experimental results verify that this 3D resonant trigger probe can be used for true 3D profile measurement.
\end{abstract}

Keywords: 3D resonant trigger probe; quartz tuning fork; interfacial force; resonant vibration; micro/nano CMM; 3D profile measurement

\section{Introduction}

In recent years, micro coordinate measuring machine (CMM) has become a research hotspot in the field of nanometer-measuring technology. Micro-CMM is a universal and primary tool used in true 3D nano-measurement of micro-devices, such as micro-electro-mechanical systems, ultra-precision mechanical components, and ultra-precision optical devices. The 3D nano-trigger technology is a key point in micro-CMM.

As a key element of micro-CMM, nano-probes are being developed on the basis of different operating principles. Rigid contact method is one of the most commonly used methods. For example, the probe based on capacitive sensors developed by NPL [1] has a resolution of $3 \mathrm{~nm}$ and probing force of $0.2 \mathrm{mN}$. The deflections of the flexures are measured by capacitance sensors inside this probe. The probe based on inductive sensors developed by METAS [2,3] has a resolution of less than $1 \mathrm{~nm}$ and probing forces of less than $0.5 \mathrm{mN}$. The translational motion is separated into its XYZ components, which are measured by three inductive sensors. A silicon-based nano-probe designed by Eindhoven University of Technology $[4,5]$ has a resolution of about $1 \mathrm{~nm}$ and probing force of less than $1 \mathrm{mN}$. 
The probe stylus is attached to three silicon elastic elements with embedded piezoresistive strain gauges for sensing the deflection. A tactile-optical fiber probe used by PTB and TU Braunschweig [6-8] has a resolution of about $1 \mathrm{~nm}$. Different from the previous deflection detection methods, the deflection of the fiber probe in the $X$ and $Y$ directions is detected by the camera system. Owing to the low stiffness of the optical fiber and the special detection method, the probing force is approximately $1 \mu \mathrm{N}$ to $100 \mu \mathrm{N}$. This fiber probe can only achieve 2D measurement. The high aspect ratio fiber probe based on a fiber Bragg grating (FBG) developed by the Harbin Institute of Technology can achieve 3D measurement with a radial resolution of $5 \mathrm{~nm}$ and an axial resolution of $8 \mathrm{~nm}[9,10]$. A touch-trigger probe developed by HFUT [11] has a repeatability of better than $5 \mathrm{~nm}$. A long-stroke 3D contact scanning probe developed by HFUT $[12,13]$ has a contact force gradient within $0.5 \mathrm{mN} / \mu \mathrm{m}$, a measuring range of $20 \mu \mathrm{m}$, and a measurement standard deviation of $30 \mathrm{~nm}$. In these contact probes mentioned above, the stiffness of the elastic elements should be carefully designed because of the mechanical deformation of the stylus and the damage of the sample surface caused by probing force.

To avoid this contradiction, some probes based on non-contact triggering methods have been proposed. Optical method is one of the representatives, but it is easily affected by the characteristics of the sample surface (such as color, roughness, reflectivity, etc.). For example, the ViScan optical probe system-which is composed of a camera sensor and an objective lens-has a measurement repeatability of less than $200 \mathrm{~nm}$ [14]. The laser-trapper probe [15-17] developed by Osaka University has a vertical resolution of about $10 \mathrm{~nm}$. Besides optical methods, many novel probes have also been developed. For example, a probing system based on a spherical capacitive sensor [18] was proposed by Harbin Institute of Technology. This probe can achieve 3D non-contact probing with resolution better than $5 \mathrm{~nm}$. The use of the non-contact method is becoming increasingly popular owing to the fast measurement speed and no contact force.

However, non-contact probes are unable to offer high resolution and accessibility to the contact one. To reduce the contact probing force, a vibrating tactile probe $[19,20]$ consisting of a triskelion device and a micro-stylus was developed by NPL, and has a probing force of nearly zero. A new variable stiffness probing system [21] designed by the University of Nottingham has the overall uncertainty of $58 \mathrm{~nm}$ for three-dimensional displacement measurement. This novel probe allows two probe stiffness values to be defined and switched between. A micro-roughness probe with ultrasonic sensor [22] developed by Mitutoyo Research Center Europe can achieve $1 \mathrm{~nm}$ resolution with the measurement force of $1 \mu \mathrm{N}$. This probe can only detect the vertical variation in the $Z$ direction in tapping mode, owing to the limitation of its tip shape. Ilmenau University of Technology has developed a resonant uniaxial microprobe [23] for the 3D measurement of small structures with a high aspect ratio. However, the oscillation amplitude of the probe is approximately $5 \mu \mathrm{m}$, which may limit the usage of the probe. A shear-mode micro-probe proposed by Tohoku University is a vibrating type and is very sensitive, but it is asymmetric in the lateral direction $[24,25]$.

The present study proposes a new probe called a 3D resonant triggering probe based on a quartz tuning fork, which is different from the previously mentioned operating principles. New mechanical models are derived and thoroughly described in this paper. The probe structure and the test results are also presented.

\section{Dynamic Contact Models and Simulation}

\subsection{Mechanical Models}

So far, the dynamic characteristics of atomic force microscopy (AFM) have been well investigated, and mechanical models between probe tip and sample surface in the vertical direction have been established and analyzed [26]. Similar to the operating mechanism of AFM, scanning probe microscopy (SPM) based on a quartz tuning fork has been proposed $[27,28]$. However, the AFM only detects the microstructure in the vertical direction. Although AFM scans along the lateral direction, it cannot detect the microstructure in that direction. In the present paper, a 3D triggering method based on 
the characteristics of a resonant device is proposed to detect the sample surface in three dimensions. This method is based on the principle that the vibration parameters of resonant devices are extremely sensitive to external microforces.

Different from conventional trigger probes (e.g., mechanical contact modes and optical noncontact modes), the probe in this study makes use of the tapping mode (TM) in contact with the sample surface in the $Z$ direction, whereas it performs in friction mode (FM) in the $X$ and $Y$ directions, as shown in Figures 1a and 2a. The mechanical models of the present study's probe in vertical ( $Z$ direction) and lateral directions ( $X$ and $Y$ directions) are established and analyzed with reference to the existing AFM mechanical models.

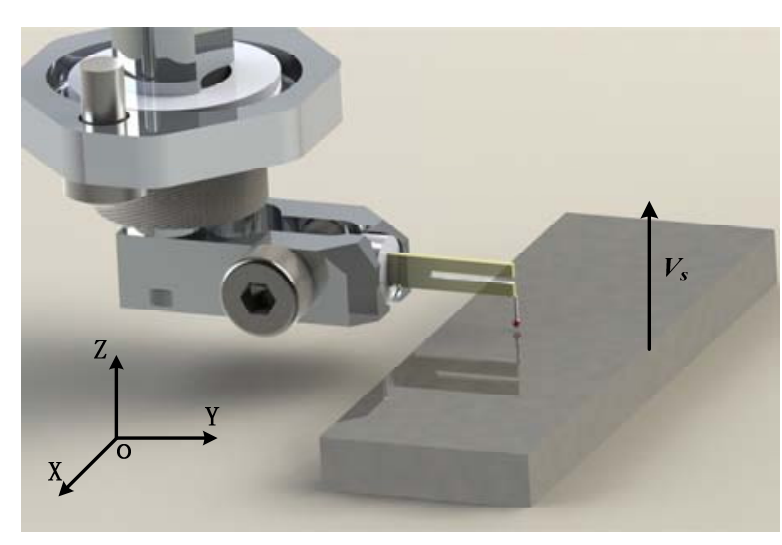

(a)

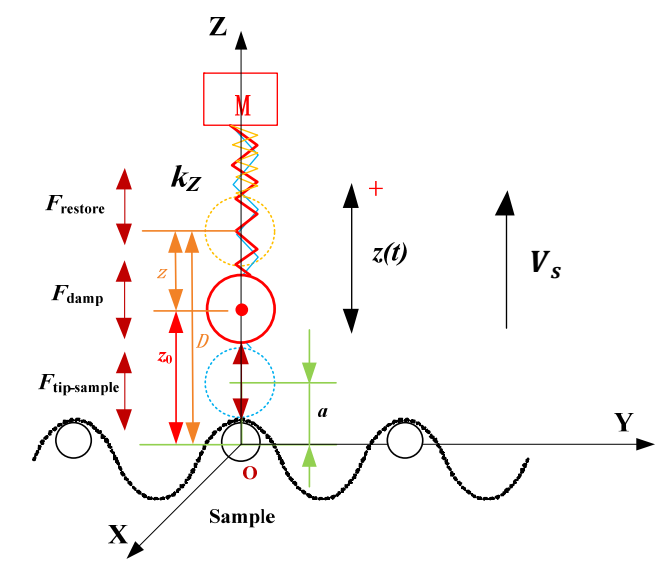

(b)

Figure 1. Tapping mode in the $\mathrm{Z}$ direction: (a) Contact model between the probe tip and the sample; (b) Schematic of the equivalent microkinetic model.

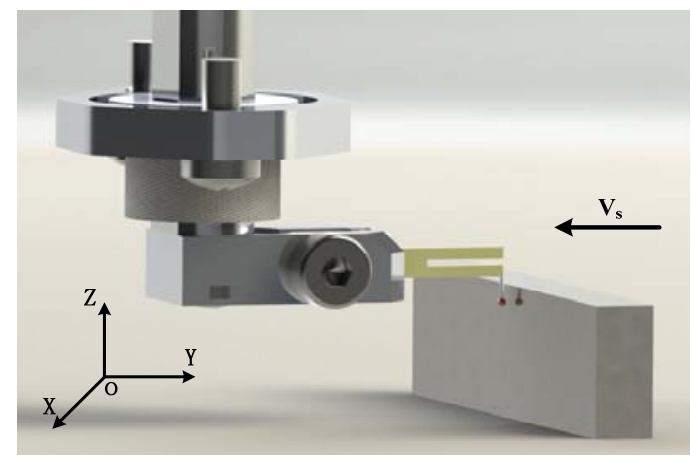

(a)

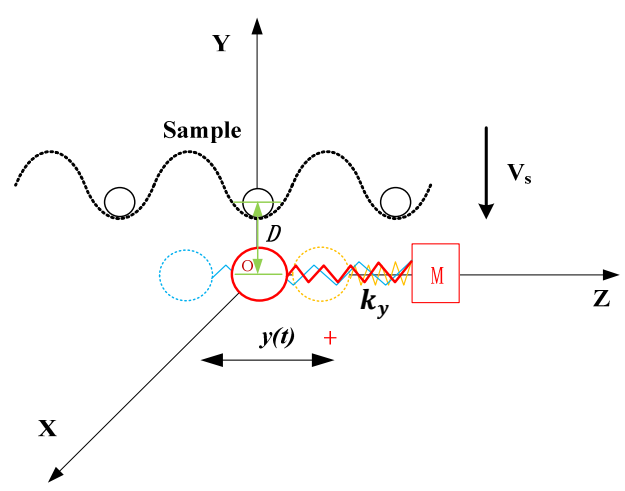

(b)

Figure 2. Friction mode in the $Y(X)$ direction: (a) Contact model between the probe tip and the sample; (b) Schematic of the equivalent microkinetic model.

At nanoscale, the interaction between the stylus microsphere tip and the sample surface is a complex process. Different interfacial forces, such as capillary, van der Waals, electrostatic, interfacial friction, and hydrogen bonding forces, influence the interaction [29]. In our previous study [30], we established a simple mass-spring contact mechanical model between the microsphere of the probe and the sample surface in three directions, and the microforces mentioned were only analyzed qualitatively and schematically. To complete the study, surface forces (e.g., van der Waals and capillary forces) and their resultant force are analyzed based on this mechanical model and interfacial tribology. The interfacial adhesive force caused by the resultant force and the contact interfacial friction force 
caused by this interfacial adhesive force are deduced. In view of the interfacial tribology, a new modified dynamic contact model is established.

The motion equations are established based on the assumption that the center of the microsphere is the centroid ( $m_{x}$ is the effective mass) and the influencing factors of force equilibrium are considered, such as damping force $\left(F_{\text {damp }}\right)$, elastic restoring force $\left(F_{\text {restore }}\right)$, and driving force $\left(F_{\text {drive }}\right)$ of the probe, as well as the effect of microforces, such as van der Waals force $\left(F_{\mathrm{vdw}}\right)$ and capillary force $\left(F_{\mathrm{cap}}\right)$, amongst others.

In the $Z$ direction, the proposed 3D trigger probe makes contact with the sample surface in TM, similar to the conventional AFM model [31]. Figure 1a shows the schematic illustration in the $Z$ direction. The probe stays still and vibrates along the $Z$ direction at its resonant frequency, while the sample is driven upward with a constant speed $\left(V_{s}\right)$. Under the TM condition, the microsphere tip maintains in normal contact with the sample surface. However, a small angular variation may exist in the real experiment condition. The existence of the average tip-sample angle may theoretically generate a lateral force. According to previous research [32], when the angle is small, the lateral force may affect the model slightly. The tapping contact between the microsphere tip and sample surface is considered to be within the scope of the elastic contact. The mechanical model is equivalent to the mass-spring coupling system of the conventional optical lever model in AFM. In Figure 1b, $z(t)$ represents the motion trail of the effective centroid, which is elastically coupled to the body $\mathrm{M}$ by a spring (equivalent spring constant $k_{z}$ ) in the $Z$ direction. $D$ represents the distance between the effective centroid and the sample surface $\left(D=z+z_{0}\right), V_{s}$ represents the approaching speed of the sample, and $a$ represents the intermolecular distance between the probe and the sample surface. Restoring force $\left(F_{\text {restore }}\right)$ is caused by the elastic deformation during the movement of the probe. Damping force $\left(F_{\text {damp }}\right)$ includes the air damping generated during the movement of the whole probe system and the energy dissipation in making contact with the sample surface. Tip-sample forces $\left(F_{\text {tip-sample }}\right)$ are those that exist between the microsphere tip and the sample, such as van der Waals force, capillary force, and so on. The arrows beside those forces in Figure $1 \mathrm{~b}$ represent the direction of the forces. Both the approach and retract processes of the microsphere interacting with the sample in this mode are divided into two phases (i.e., $D>a$ and $D \leq a$ ), because interfacial forces are associated with the distance between the microsphere tip and sample surface. In the $D>a$ phase, the interfacial force is mainly represented by the long-range van der Waals attractive force $\left(F_{\text {att }}\right)$, whereas in the $D \leq a$ phase, the interfacial force is mainly represented by surface adhesion ( $F_{\text {ad }}$; Derjaguin-Muller-Toporov (DMT) theory) [33] and short-ranged repulsive force $\left(F_{\text {rep }}\right)$. Therefore, the initial motion equation in the $Z$ direction is established as:

$$
\begin{cases}m_{z} \ddot{z}(t)+\beta \gamma \dot{z}(t)+\mu z(t)=F_{\text {drive }}-\frac{H R}{6\left(z+z_{0}\right)^{2}}+2 \pi r \gamma_{\mathrm{Lg}} \alpha\left(\cos \theta_{1}+\cos \theta_{2}\right) & \text { for } D>a \\ m_{z} \ddot{z}(t)+\beta \gamma \dot{z}(t)+\mu z(t)=F_{\text {drive }}-\frac{H R}{6 a^{2}}+\frac{4}{3} E^{*} \sqrt{R}\left(a-z_{0}-z\right)^{3 / 2}+2 \pi r \gamma_{\mathrm{Lg}} \alpha\left(\cos \theta_{1}+\cos \theta_{2}\right) & \text { for } D \leq a\end{cases}
$$

where $\gamma$ is the general damping coefficient ( $\beta$ is a constant, let $\beta=1$ ), $\mu$ is the gradient of the general elastic restoring force (which is the equivalent elastic stiffness $k_{z}$ ), $H$ is the Hamaker constant, $R$ is the microsphere radius, $z_{0}$ is the resonant center of the microsphere, $z$ is the instantaneous tip position, $r$ is the sphere-sample surface contact radius, $\gamma_{\mathrm{Lg}}$ is the surface free energy of water (steam pressure), $\theta_{1}$ is the contact angle between microsphere and water film, $\theta_{2}$ is the contact angle between sample surface and water film, $\alpha$ is the coefficient related to the surface tension, $F_{\text {drive }}$ is the driving force, and $E^{*}$ is the effective Young's modulus.

In the $X$ and $Y$ directions, such as the situation in the XOZ plane, the microsphere tip makes contact with the sample surface along the $Y$ direction, as shown in Figure 2a. For convenience, the equivalent movement model in the $\mathrm{XOZ}$ plane is built by rotating the entire initial model by $90^{\circ}$ counterclockwise, as shown in Figure 2b. According to nanotribology, adhesion and applied loads are in the same order of magnitude under nanofriction conditions. In other words, because of the existence of interfacial adhesion, generalized Hertz friction theory is not fully applicable to the present study. Considering the lateral contact stiffness and viscous stiffness, the mechanical model is equivalent to the model shown in 
Figure $2 \mathrm{~b}$, where $y(t)$ represents the motion trail of the effective centroid, which is coupled elastically to the body $M$ by a spring with an equivalent spring constant $k_{y}$ in the $Y(X)$ direction, $V_{s}$ represents the approaching speed of the sample, and $a$ represents the intermolecular distance. Based on the model by Zwörner [34], the dynamic analysis is presented after full consideration of the stress condition where the microsphere tip is in contact with the sample surface. In addition, according to the wetting phenomenon [35] in nanotribology and the continuum medium principle, the water film with a certain thickness (about several nanometers) may be formed in microscale within the contact area because of the air humidity and the hydrophilic nature of the sample surface. Therefore, the initial motion equation in the $Y(X)$ direction is expressed as:

$$
m_{y} \ddot{y}(t)+\beta \gamma \dot{y}(t)+\mu^{\prime} y(t)=F_{\text {drive }}-\frac{K r^{3}}{2 R}-\tau_{c} A-V-2 \pi r \gamma_{\mathrm{Lg}} \alpha\left(\cos \theta_{1}+\cos \theta_{2}\right)
$$

where $y(t)$ is the motion of the effective centroid, $\gamma$ is the general damping coefficient ( $\beta$ is a constant, let $\beta=1$ ), $\mu^{\prime}$ is the gradient of the general elastic restoring force (which is the equivalent elastic stiffness $\left.k_{y}\right), R$ is the microsphere radius, $K$ is the equivalent elastic constant modulus associated with the material, and $K=4 / 3 E^{*}, E^{*}$ is the equivalent Young's modulus, $r$ is the sphere-sample surface contact radius, $\tau_{c}$ is the shear stress acting on the adhesive contact area $A, V$ indicates the van der Waals force in the $Y$ direction, $\gamma_{\mathrm{Lg}}$ is the surface free energy of water (steam pressure), $\theta_{1}$ is the contact angle between microsphere and water film, $\theta_{2}$ is the contact angle between sample surface and water film, and $\alpha$ is the coefficient related to the surface tension.

In Equations (1) and (2), $\beta \gamma \dot{z}(t)$ and $\beta \gamma \dot{y}(t)$ represent the damping force $\left(F_{\text {damp }}\right), \mu z(t)$ and $\mu^{\prime} y(t)$ represent the elastic restoring force $\left(F_{\text {restore }}\right), 2 \pi r \gamma_{\mathrm{Lg}} \alpha\left(\cos \theta_{1}+\cos \theta_{2}\right)$ is the capillary force $\left(F_{\text {cap }}\right)$, $F_{\text {att }}=-H R /\left[6\left(z+z_{0}\right)^{2}\right]$ is the long-range van der Waals force in the $Y(X)$ direction, and $F_{\mathrm{ad}}=-H R /\left(6 a^{2}\right)$ and $F_{\text {rep }}=4 / 3 E^{*} \sqrt{R}\left(a-z_{0}-z\right)^{3 / 2}$ are the adhesive force and short-range repulsive force, respectively. In Equation (2), $F_{\mathrm{ad}}=S=\left(\mathrm{Kr}^{3}\right) / 2 R$ and $F_{\text {fri }}=\tau_{c} A$ indicate the adhesive force and interfacial friction force, respectively.

The interfacial adhesive force is the dominant factor for interfacial friction during the dynamic contact process of the sphere-sample interaction in the friction model in the $Y(X)$ direction; this force is generated by co-action of several microforces [30] and becomes the "needle point force field" within the nanocontact area. In the nanometer scale, the friction coefficient is no longer a constant value, and the relationship between interfacial friction $\left(F_{\text {fric }}\right)$ and normal pressure $\left(F_{\mathrm{N}} ;\right.$ namely, $\left.F_{\mathrm{ad}}\right)$ is nonlinear. Their relationship in the nanometer scale is regarded as [36]:

$$
F_{\text {fric }}=\zeta F_{\mathrm{N}}^{2 / 3}=\zeta\left(\frac{K a^{3}}{2 R}\right)^{2 / 3}=\zeta\left(6 \pi R \gamma_{\mathrm{SV}}\right)^{2 / 3} \propto r_{12}{ }^{-3 / 4}
$$

where $\zeta$ is a system parameter similar to the friction coefficient, $K$ is the equivalent elastic constant associated with the material, $R$ is the probe microsphere radius, $a$ is the contact radius, $\gamma_{\mathrm{SV}}$ is the interface energy in the contact areas between the microsphere and the sample surface, and $\gamma_{\mathrm{SV}}=H /\left(24 \pi r_{12}{ }^{2}\right), r_{12}$ is the distance between them. According to the interfacial tribology, the motion model in the $Y(X)$ direction is modified into the motion equation of the equivalent centroid of the $3 \mathrm{D}$ resonant trigger probe under damping vibration as follows:

$$
m_{y} \ddot{y}(t)+\frac{m_{y} \omega_{0}}{Q} \dot{y}+k y=k A_{0} \cos (\omega t)+\zeta\left(\frac{R H}{4 r_{12}{ }^{2}}\right)^{\frac{2}{3}} \operatorname{sign}(\dot{y})
$$

where $\omega_{0}$ is the resonant frequency of the probe system, $Q$ is the quality factor, $k$ is the equivalent force constant, $H$ is the Hamaker constant, and $A_{0}$ and $\omega$ are the amplitude and angular frequency of the driving force, respectively. The driving force is expressed by $F_{\text {drive }}=k A_{0} \cos (\omega t)$. 


\subsection{Microforces and Mathematical Simulation}

As the main cause of interfacial friction, the interfacial adhesive force is resulted from the co-action of the van der Waals force, capillary force, and other microforces. These forces should be analyzed in order to understand the mechanical properties and triggering mechanism in three dimensions.

The van der Waals force is a type of electromagnetic force that has neither directivity nor saturability, and has an effective range from nanometer to sub-nanometer. It mainly arises from three kinds of forces with different generating mechanisms: orientation force between polar molecules, induction force between polar and nonpolar molecules, and dispersion force among nonpolar molecules. The dynamic contact model of the sphere-sample is analyzed based on the Hamaker hypothesis using the continuum medium method. Assuming that the sample surface is an infinite plane, the van der Waals force $\left(F_{\mathrm{vdw}}\right)$ between the microsphere and the sample surface is calculated as follows [37]:

$$
F_{\mathrm{vdw}}=\frac{T R}{180 D^{8}}-\frac{H R}{6 D^{2}}
$$

where $R$ is the microsphere radius, $D$ is the distance between the microsphere and the sample surface; $T$ is the expression of $\pi^{2} \rho_{1} \rho_{2} A ; H$ is the expression of $\pi^{2} \rho_{1} \rho_{2} B$, where $\rho_{1}$ and $\rho_{2}$ are the densities of the microsphere and the sample surface, respectively; $A$ is the repulsive force constant; and $B$ is the attractive force constant.

When the absolute distance $(D)$ between the microsphere and the sample surface is considered as a variable, the change trend of the van der Waals force in the microcontact areas are obtained through simulation. Let $R=80 \mu \mathrm{m}$, and the simulation of the van der Waals force is obtained as shown in Figure 3. Here, the horizontal axis represents the distance between microsphere tip and sample surface, and the longitudinal axis represents the magnitude of van der Waals force. The van der Waals force in section A behaves as a repulsive force. By contrast, the van der Waals force in section B behaves as an attractive force. In other words, when the microsphere approaches the sample surface, the van der Waals force changes the "needle point force field" from a long-range attractive force to a short-range repulsive force in the microcontact regions.

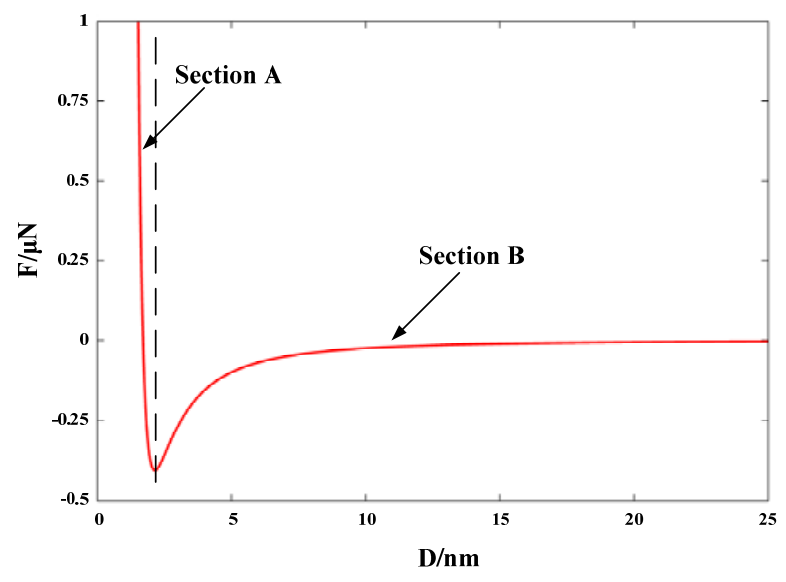

Figure 3. Simulation of the van der Waals force.

Capillary force $\left(F_{\text {cap }}\right)$ is the surface tension caused by the wetting action of the water film between sample surface and microsphere. The water film is generated from the air humidity and hydrophilicity of the sample surface [38]. Figure 4 shows the diagram of the capillary force existing in nanocontact areas between microsphere and sample surface, where $R$ is the radius of the microsphere, $D$ is the distance between the microsphere and the sample surface, $r$ is the curvature radius of the water film, 
$\theta_{1}$ is the contact angle between the microsphere and the water film, and $\theta_{2}$ is the contact angle between the sample surface and the water film. When $R \gg D$, the following is obtained:

$$
F_{\text {cap }} R \gg D=2 \pi R \gamma_{\text {Lg }}\left(\cos \left(\theta_{1}+\beta\right)+\cos \theta_{2}-\frac{D}{r}\right) \approx 4 \pi R \gamma_{\mathrm{Lg}}
$$

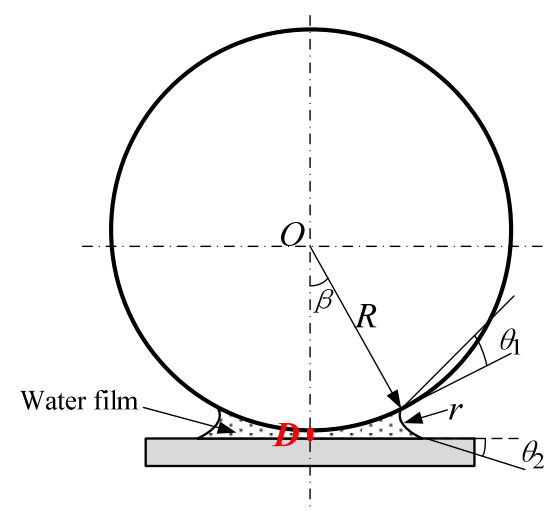

Figure 4. Capillary force between the microsphere and the plane surface.

Under the micro-angle condition, the capillary force and the interfacial friction in the modified model (shown in Equation 3) have the same order of magnitude, which means that under certain air humidity, the capillary force may multiply the resultant force of the system (items on the right side of Equations (1) and (2), except $F_{\text {drive }}$ ). This finding proves that, under the atmospheric environment, the influence of capillary force on the dynamic contact model of this probe system cannot be ignored.

As main microforces in the mesoscopic scale, the co-action of the van der Waals and capillary forces determines the dynamic mechanical properties of nanocontact between the microsphere tip and the sample surface. According to the analysis of the established models, van der Waals force is invalid outside the nanocontact region, and interfacial adhesion and interfacial friction are inexistent. The probe microsphere maintains a resonant vibration with constant amplitude because no external force acts on it. Figure 5 shows a simulation diagram of the probe's FM vibration amplitude in the $Y(X)$ direction. The horizontal axis represents the distance between the microsphere tip and the sample surface, and the longitudinal axis represents the amplitude of the microsphere. When the tip-sample interaction comes into the nanocontact region, the amplitude significantly attenuates, and the resonance frequency shifts with the change of the force gradient between the probe microsphere and the sample surface. On the basis of this phenomenon, this probe can be used as a 3D-positioning trigger sensor.

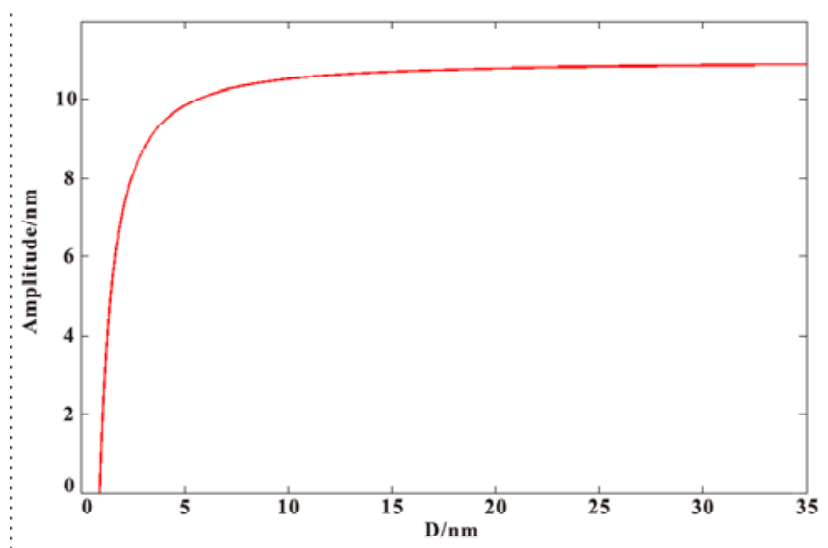

Figure 5. Simulation of amplitude versus tip-sample distance in the $Y(X)$ direction. 


\section{Experiments on the 3D Quartz Tuning-Fork Probe}

\subsection{Probe System}

A 3D resonant positioning trigger probe using a quartz tuning fork is fabricated to verify the reliability of the micromechanism of the dynamic contact model and the feasibility of the positioning trigger method mentioned. The quartz tuning fork has the advantage of a stable mechanical resonant characteristic, especially a high-quality factor, which is approximately $10^{3}-10^{5}$ [39]. The higher quality factor may contribute to the sensitivity of the device. Therefore, the material has been used to fabricate scanning probes for 2D measurement [24]. However, in the current study, the probe based on the quartz tuning fork is further used for 3D triggering. While the quartz tuning fork vibrates in its resonant state, its resonant vibrating parameters (resonant vibrating amplitude and resonant frequency) are extremely sensitive to external microforces. Figure 6 shows a typical frequency spectra of the 3D trigger probe in air. The horizontal axis represents the excitation frequency of the microsphere, and the longitudinal axis represents the amplitude voltage of the microsphere. The figure shows that the quality factor is about 3200 , which is deemed very high.

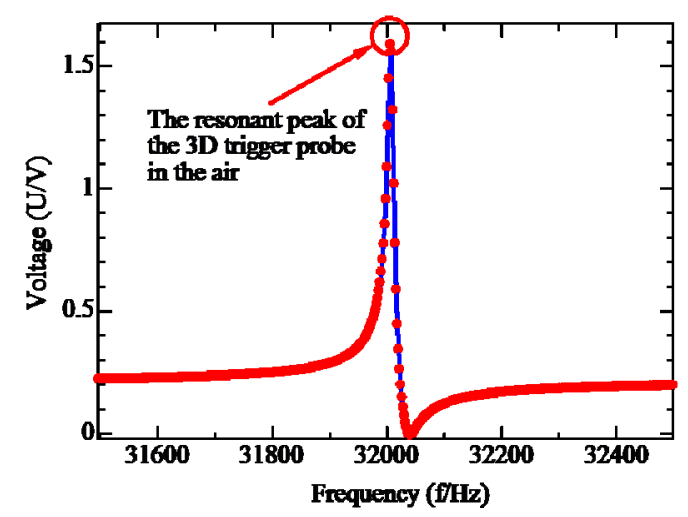

Figure 6. Typical frequency spectra of the 3D trigger probe in air.

Figure 7 shows the structure of the probe system. The base of the quartz tuning fork is fixed steadily on the probe frame to avoid additional energy loss when the probe vibrates. One of the quartz tuning fork arms serves as a piezoelectric driver, and the other serves as a piezoelectric sensor to output the electrical signal. A home-made integrated fiber microstem with a microsphere tip is mounted on the free end of the sensing arm. The length of the stem is about $1 \mathrm{~mm}$, and the diameter of the microsphere is $80 \mu \mathrm{m}$ [40]. During the operation, the probe is driven in resonant state by a sinusoidal signal. When the microsphere tip approaches the sample surface, the amplitude attenuates, and the resonant frequency shifts significantly as soon as the tip-sample interaction comes into the nanocontact region. As a result, the electrical output signal is changed. This signal can be used as the trigger signal, but it cannot distinguish the contact direction. For micro/nano CMM, the unknown contact directions may bring ambiguities when reconstructing a surface. However, in most cases, the basic profiles of the samples are already known, according to the blueprints or observation. So, the contact directions can be predicted when measuring these objects. 


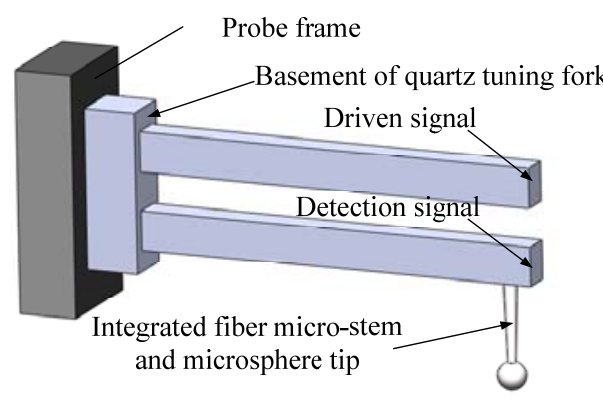

(a)

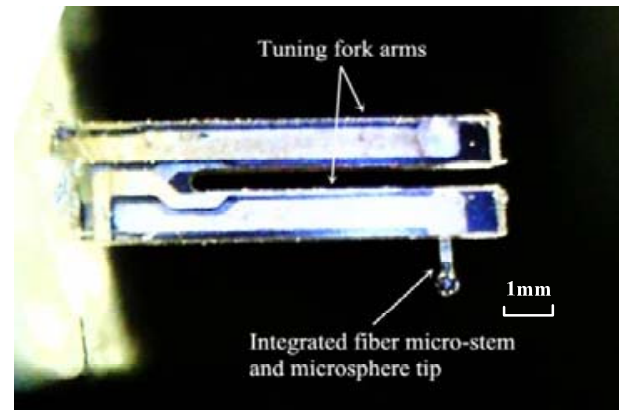

(b)

Figure 7. (a) Schematic diagram of the structure of the probe system; (b) Enlarged picture of the quartz tuning fork and the integrated fiber microstem and microsphere tip.

\subsection{D Trigger Positioning System}

Based on the mentioned quartz tuning fork probe combined with a 3D nanopositioning stage, a feedback control module, and a signal processing circuit, a 3D trigger positioning system is constructed, as shown in Figure 8. The quartz tuning fork probe keeps vibrating at its resonant frequency. The amplitude of the probe is detected by the amplitude feedback circuit. When the probe tip contacts the sample surface in a certain direction, the vibrating amplitude of the probe attenuates because of the force interaction between the probe tip and the sample surface. The phase feedback circuit using Phase Locked Loop (PLL) is also designed as an alternative for the system. To verify the amplitude-distance model that we established, only the amplitude feedback circuit is used in the experiment part. The 3D nanopositioning stage used in this study is produced by Physik Instrumente Co., Ltd (Karlsruhe, Germany). The nanostages in the $Y(X)$ and $Z$ directions are P-611.2S and P-753.1CD, respectively. The $X(Y)$ stage has a closed-loop resolution of $2 \mathrm{~nm}$ and a positioning repeatability of less than $10 \mathrm{~nm}$. The $Z$ stage has a closed-loop resolution of $0.05 \mathrm{~nm}$ and positioning repeatability of $\pm 1 \mathrm{~nm}$.

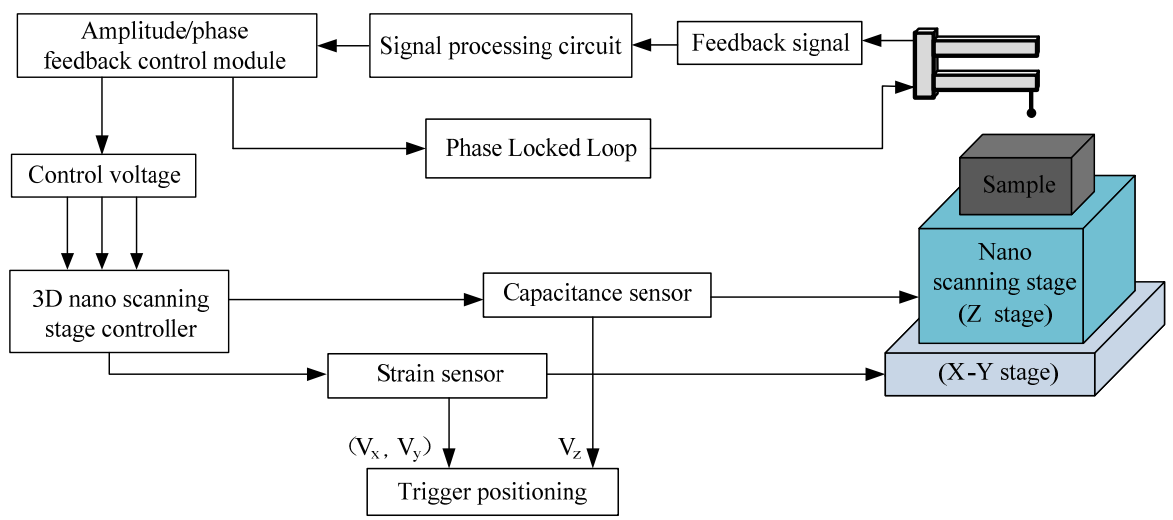

Figure 8. Schematic of the experimental system for the quartz tuning fork.

\subsection{Experimental Results}

Noise level, sensitivity, trigger resolution, probing force, and unidirectional repeatability are all important parameters used to evaluate the quality of a positioning trigger probe. In our previous research [30], the working surface of an ultra-precision gauge block was used as the target to obtain the approaching curves of the probe in the $X, Y$, and $Z$ directions (the approaching curve shows the relationship between the vibrating amplitude of the probe and the displacement of the stage). In Figure 9, when the probe is in the nanocontact area, the sensitivity of the tip-sample-which is 
determined by the curve slope of the linear segment obtained through the linear regression method-is $2.62 \mathrm{~V} / \mu \mathrm{m}$ in the $X$ direction. The trigger resolution of $0.38 \mathrm{~nm}$ is determined via the system noise level of $1 \mathrm{mV}$. In the same way, the sensitivity is $2.21 \mathrm{~V} / \mu \mathrm{m}$ and the resolution is $0.45 \mathrm{~nm}$ in the $Y$ direction. In the $Z$ direction, the sensitivity is $2.24 \mathrm{~V} / \mu \mathrm{m}$ and the resolution is $0.45 \mathrm{~nm}$. The results show that this probe has a sensitivity of less than $0.5 \mathrm{~nm}$ in all directions. This probe is proven to have sub-nanometer resolution.

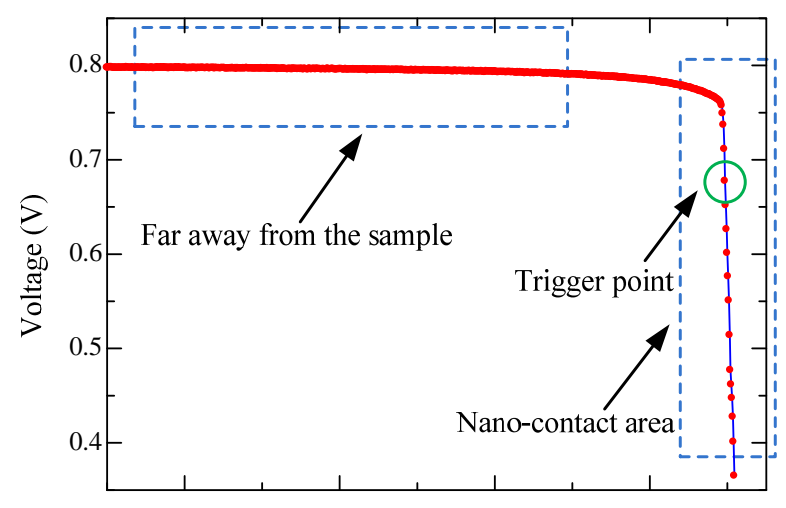

Relative displacememt (1 micrometer/div.)

Figure 9. Approaching curve in the $X$ direction.

Figure 9 shows the approaching curve in the $X$ direction. The horizontal axis represents the relative displacement that the positioning stage moves, and the longitudinal axis represents the amplitude voltage of the microsphere. Before the nanocontact regions, the microsphere of the probe remains resonant with constant amplitude. When the tip-sample interaction goes into the repulsive region of section A in Figure 3, the amplitude significantly attenuates because of the interfacial resultant force of "needle point force field" - a result that is consistent with the model analyzed. This finding indicates that the proposed model qualitatively explains the interaction between the microsphere of the $3 \mathrm{D}$ resonant trigger probe and the sample surface. When the oscillation amplitude of the probe decreases to 80 percent of the initial value, this position of the nanostage is recorded as a trigger point. The probing force of the system is also calculated. In the lateral direction, assuming that the contact between the microsphere and the sample surface is rigid, the contact force can be calculated simply by the blending force of the micro-stem. In Figure 9, the distance between the start-decreasing point and the trigger point represents the blending distance (over travel) of the micro-stem which is less than $50 \mathrm{~nm}$. With the stiffness of $143 \mathrm{~N} / \mathrm{m}$ of the micro-stem, the calculation of the probing force is about $7 \mu \mathrm{N}$. In the $Z$ direction, the microsphere is in contact with the sample surface in tapping mode, which is not rigid contact. Besides, the approaching speed is in sub micrometer per second, which is very low. So, according to the model we established, the probing force in the $Z$ direction is in a similar magnitude to the van der Waals force.

Repeatability is an important evaluation parameter of a trigger positioning system. Several factors may influence repeatability, such as voltage fluctuations caused by unstable factors of the driving signal, Abbe error resulting from the mechanical structure and positioning accuracy of the nanostage in the process of testing, changes in environmental conditions, external vibration, and other random errors. To verify the reliability of the probe system, the repeatability of the triggering process of the probe has been evaluated by approaching the probe repeatedly at the same measurement point in the working surface of an ultra-precision gauge block. Figure 10a shows the repeatability tests for 10 times in the $X, Y$, and $Z$ directions. The vertical axis indicates the offsets from the average displacement value of the trigger point. The limit error is $23 \mathrm{~nm}$ in the $X$ direction, $23 \mathrm{~nm}$ in the $Y$ direction, and $12 \mathrm{~nm}$ in the $Z$ direction. The standard deviation of the $X, Y$, and $Z$ directions is $7.53 \mathrm{~nm}, 8.56 \mathrm{~nm}$, and $4.12 \mathrm{~nm}$ respectively. Figure $10 \mathrm{~b}$ shows the unidirectional approaching curves of the probe in an arbitrary 
direction. The horizontal axis represents the relative displacement that the positioning stage moves, and the longitudinal axis represents the amplitude voltage of the microsphere. Ten voltage curves are measured repeatedly in this direction, and the measuring time is about two minutes. The experimental results illustrate that the unidirectional repeatability error in an arbitrary direction in three dimensions is in the nanometer order of magnitude, with maximum about $40 \mathrm{~nm}$. The probe is proven to have the ability for trigger positioning with high stability.

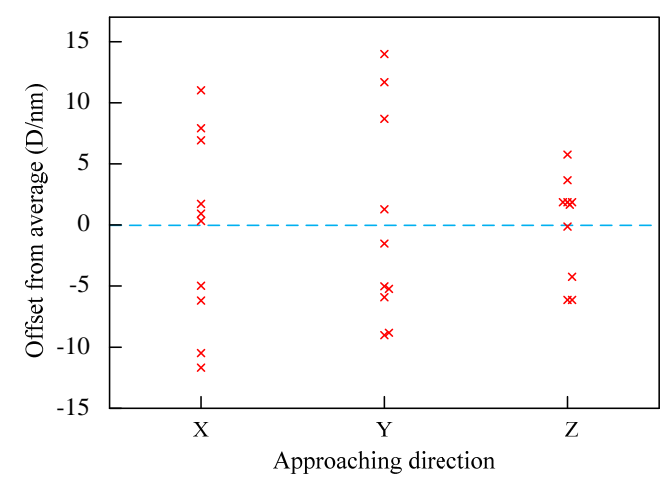

(a)

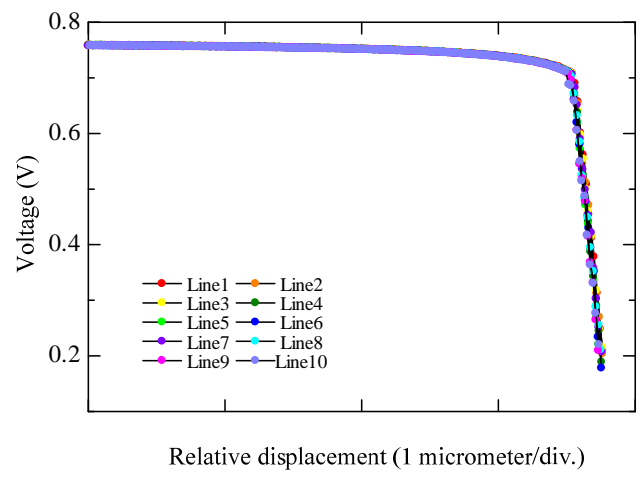

(b)

Figure 10. (a) Triggering repeatability evaluation in the $X, Y$, and $Z$ directions. (b) Unidirectional repeatability curves in an arbitrary direction.

In fact, most of the repeatability errors mentioned above are due to the positioning repeatability error of the stage and the random angular error of the stage magnified by Abbe offset of the experimental system, rather than the probe itself. The $X(Y)$ positioning stage and the $Z$ positioning stage are two separate parts. As shown schematically in Figure 8, the $Z$ stage is stacked on the $X(Y)$ stage. The sample is located on the top of the $Z$ stage and moves with it. A certain distance exists between the $X / Y$ stage of the 3D nanopositioning stage and the probe tip. This distance implies a large Abbe offset in the $X(Y)$ direction between the measurement point (tapping point of the microsphere tip of the probe) and the $X / Y$ reference length scales (strain sensor) inside the $X / Y$ stage. In the $Z$ direction, an Abbe offset also exists between the measurement point and the $Z$ reference length scale (capacitance sensor) inside the $Z$ stage. In other words, limited by the mechanical structure of the system, Abbe errors exist in all three directions. If the experimental system is modified and the Abbe offsets are zero, then the repeatability error of the probe will improve significantly.

\section{Summary}

The mechanical models of the interactions between the microsphere tip of the probe and the sample surface are constructed based on interfacial force theory, and the dynamic contact mechanism in the $X(Y)$ and $Z$ directions are analyzed. The combined action is presented according to the detailed analysis of the mechanism of microforces (van der Waals and capillary forces) in the mesoscopic scale. As the distance between the microsphere of the probe and the sample surface decreases, the resultant force changes from a long-range attractive force to a short-range repulsive force, the amplitude of the probe significantly attenuates, and then the resonant frequency shifts because of the changing force gradient. Although the trigger signal cannot distinguish the contact direction, it has little influence when measuring the objects with clear structures. Moreover, a visual guide system will be used to solve this problem and improve the measuring speed in the future.

Through experiments, the trigger resolution of the resonant trigger probe proposed in three dimensions is found to achieve a sub-nanometer order of magnitude, and the unidirectional repeatability error is approximately $40 \mathrm{~nm}$. The results indicate that the $3 \mathrm{D}$ resonant trigger probe 
system constructed in this study achieves the function of nanoscale positioning with high feasibility and reliability, and that it can be used as a trigger probe for micro-CMM.

Acknowledgments: This study is sponsored by the National Natural Science Foundation of China (No. 51475131).

Author Contributions: Qiangxian Huang conceived and wrote the paper. Chen Chen established and analyzed the Mechanical model of the probe system. Kui Wu, Lianshen Zhang and Ruijun Li contributed mainly to the control system and experiments. Kuang-Chao Fan guided the experiment design and revised the paper.

Conflicts of Interest: The authors declare no conflict of interest.

\section{References}

1. Peggs, G.N.; Lewis, A.J.; Oldfield, S. Design for a compact high-accuracy CMM. CIRP Ann. 1999, 48, 417-420. [CrossRef]

2. Meli, F.; Fracheboud, M.; Bottinelli, S.; Bieri, M.; Thalmann, R.; Breguet, J.M.; Clavel, R. High precision, low force 3D touch probe for measurements on small objects. In Proceedings of the EUSPEN, International Topical Conference, Aachen, Germany, 19-20 May 2003.

3. Küng, A.; Meli, F.; Thalmann, R. Ultraprecision micro-CMM using a low force 3D touch probe. Meas. Sci. Technol. 2005, 18, 319-327. [CrossRef]

4. Haitjema, H.; Pril, W.O.; Schellekens, P.H.J. Development of a silicon-based nanoprobe system for 3-D measurements. CIRP Ann. 2001, 50, 365-368. [CrossRef]

5. Pril, W.O. Development of High Precision Mechanical Probes for Coordinate Measuring Machines. Ph.D. Thesis, Technische Universiteit Eindhoven, Eindhoven, The Netherlands, 2002.

6. Brand, U.; Kleine-Besten, T.; Schwenke, H. Development of a special CMM for dimensional metrology on microsystem components. In Proceedings of the Annual, Meeting of the ASPE, Scottsdale, AZ, USA, 22-27 October 2000; pp. 542-546.

7. Tutsch, R.; Andräs, M.; Neuschaefer-Rube, U.; Petz, M. Three dimensional tactile-optical probing for the measurement of microparts. In Proceedings the SENSOR, Nurnberg, Germany, 26 May 2009; Volume 2, pp. 139-144.

8. Petz, M.; Tutsch, R.; Christoph, R.; Andraes, M.; Hopp, B. Tactile-optical probes for three-dimensional microparts. Measurement 2012, 45, 2288-2298. [CrossRef]

9. Cui, J.W.; Li, J.Y.; Feng, K.P.; Tan, J.B.; Zhang, J. A 3D fiber probe based on orthogonal micro focal-length collimation and fiber Bragg grating. Meas. Sci. Technol. 2016, 27, 07405. [CrossRef]

10. Feng, K.P.; Cui, J.W.; Zhao, S.Y.; Li, J.Y.; Tan, J.B. A twin FBG probe and integration with a micro-holemeasuring machine for the measurement of micro holes of high aspect ratios. IEEE-ASME Trans. Mech. 2016, 21, 1242-1251. [CrossRef]

11. Li, R.J.; Xiang, M.; He, Y.X.; Fan, K.C.; Cheng, Z.Y.; Huang, Q.X.; Zhou, B. Development of a high-precision touch-trigger probe using a single sensor. Appl. Sci. 2016, 6, 86. [CrossRef]

12. Li, R.J.; Fan, K.C.; Huang, Q.X.; Zhou, H.; Gong, E.M.; Xiang, M. A long-stroke 3D contact scanning probe for micro/nano coordinate measuring machine. Prec. Eng. 2016, 43, 220-229. [CrossRef]

13. Huang, Q.X.; Wu, K.; Wang, C.C.; Li, R.J.; Fan, K.C.; Fei, Y. Development of an abbe error free micro coordinate measuring machine. Appl. Sci. 2016, 6, 97. [CrossRef]

14. Thomas, R.K.; Thom, J.H. Metrology, sensors and control. In Micromanufacturing, 1st ed.; Ehmann, K.F., Bourell, D., Eds.; Springer: Dordrecht, The Netherlands, 2007; pp. 89-109.

15. Takaya, Y.; Imai, K.; Ha, T.; Miyoshi, T. Vibrational probing technique for the Nano-CMM based on optical radiation pressure control. CIRP Ann.-Manuf. Technol. 2010, 53, 421-424. [CrossRef]

16. Michihata, M.; Nagasaka, Y.; Hayashi, T.; Takaya, Y. Probing technique using circular motion of a microsphere controlled by optical pressure for a nanocoordinate measuring machine. Appl. Opt. 2009, 48, $198-205$. [CrossRef] [PubMed]

17. Michihata, M.; Hayashi, T.; Nakai, D.; Takaya, Y. Microdisplacement sensor using an optically trapped microprobe based on the interference scale. Rev. Sci. Instrum. 2010, 81, 015107. [CrossRef] [PubMed]

18. Tan, J.B.; Cui, J.N. Ultraprecision 3D probing system based on spherical capacitive plate. Sens. Actuators A Phys. 2010, 159, 1-6. [CrossRef] 
19. Claverley, J.D.; Leach, R.K. A vibrating micro-scale CMM probe for measuring high aspect ratio structures. Microsyst. Technol. 2010, 16, 1507-1512. [CrossRef]

20. Claverley, J.D.; Leach, R.K. Development of a three-dimensional vibrating tactile probe for miniature CMMs. Precis. Eng. 2013, 37, 491-499. [CrossRef]

21. Alblalaihid, K.; Kinnell, P.; Lawes, S.; Desgaches, D.; Leach, R. Performance assessment of a new variable stiffness probing system for Micro-CMMs. Sensors 2016, 16, 492. [CrossRef] [PubMed]

22. Hidaka, K.; Saito, A.; Koga, S. Study of a micro-roughness probe with ultrasonic sensor. CIRP Ann.-Manuf. Technol. 2008, 57, 489-492. [CrossRef]

23. Goj, B.; Dressler, L.; Hoffmann, M. Semi-contact measurements of three-dimensional surfaces utilizing a resonant uniaxial microprobe. Meas. Sci. Technol. 2014, 25, 064012. [CrossRef]

24. Ito, S.; Kodama, I.; Gao, W. Development of a probing system for a micro-coordinate measuring machine by utilizing shear-force detections. Meas. Sci. Technol. 2014, 25, 064011. [CrossRef]

25. Ito, S.; Kikuchi, H.; Chen, Y.; Shimizu, Y.; Gao, W.; Takahashi, K.; Kanayama, T.; Arakawa, K.; Hayashi, A. A micro-coordinate measurement machine (CMM) for large-scale dimensional measurement of micro-slits. Appl. Sci. 2016, 6, 156. [CrossRef]

26. Giessibl, F.J. Advances in atomic force microscopy. Rev. Mod. Phys. 2003, 75, 949-983. [CrossRef]

27. Giessibl, F.J. High-speed force sensor for force microscopy and profilometry utilizing a quartz tuning fork. Appl. Phys. Lett. 1998, 73, 3956-3958. [CrossRef]

28. Tyrrell, J.W.G.; Sokolov, D.V.; Danzebrink, H.U. Development of a scanning probe microscope compact sensor head featuring a diamond probe mounted on a quartz tuning fork. Meas. Sci. Technol. 2003, 14, 2139-2143. [CrossRef]

29. Murakami, H.; Katsuki, A.; Sajima, T.; Fukuda, M. Reduction of liquid bridge force for 3D microstructure measurements. Appl. Sci. 2016, 6, 153. [CrossRef]

30. Yu, H.J.; Huang, Q.X.; Li, Z.B.; Wang, M.C.; Wei, J.P. Dynamic analysis of mechanical model for three-dimensional resonant trigger probe and experiment. In Proceedings of the SPIE, Chengdu, China, 31 January 2013.

31. García, R.; Paulo, A.S. Attractive and repulsive tip-sample interaction regimes in tapping-mode atomic force microscopy. Phys. Rev. B 1999, 60, 4961-4967. [CrossRef]

32. Moreno-Herrero, F.; Depablo, P.J.; Colchero, J.; Gómez-Herrero, J.; Baró, A.M. The role of shear forces in scanning force microscopy: a comparison between the jumping mode and tapping mode. Surf. Sci. 2000, 453, 152-158. [CrossRef]

33. Zhou, X.W.; Fang, Y.C. A virtual tapping-mode Atomic force microscope. In Proceedings of the International Conference on Nano/Micro Engineered and Molecular Systems, Zhuhai, China, 18-21 January 2006; pp. 501-504.

34. Zwörner, O.; Hölscher, H.; Schwarz, U.D.; Wiesendanger, R. The velocity dependence of frictional forces in point-contact friction. Appl. Phys. A Mater. Sci. Process. 1998, 66 (Suppl. 1), 263-267. [CrossRef]

35. Wen, S.Z. Nano Tribology; Tsinghua University Press: Beijing, China, 1998.

36. Hild, W.; Ahmed, I.U.; Hungenbach, G.; Scherge, M.; Schaefer, J.A. Microtribological properties of silicon and silicon coated with self-assembled monolayers: Effect of applied load and sliding velocity. Tribol. Lett. 2007, 25, 1-7. [CrossRef]

37. Tian, W.C.; Jia, J.Y. Amelioration of the Haymaker homogeneous material hypothesis. Acta Phys. Sin. 2003, $52,1061-1065$.

38. Butt, H.J.; Kappl, M. Normal capillary forces. Adv. Colloid Interface Sci. 2009, 146, 48-60. [CrossRef] [PubMed]

39. Ctistis, G.; Frater, E.H.; Huisman, S.R.; Korterik, J.P.; Herek, J.L.; Vos, W.L.; Pinkse, P.W.H. Controlling the quality factor of a tuning-fork resonance between 9 and $300 \mathrm{~K}$ for scanning-probe microscopy. J. Phys. D Appl. Phys. 2011, 44, 375502. [CrossRef]

40. Yu, H.J.; Huang, Q.X.; Zhao, J. Fabrication of an optical fiber micro-sphere with a diameter of several tens of micrometers. Materials 2014, 7, 4878-4895. [CrossRef]

(C) 2017 by the authors. Licensee MDPI, Basel, Switzerland. This article is an open access article distributed under the terms and conditions of the Creative Commons Attribution (CC BY) license (http:/ / creativecommons.org/licenses/by/4.0/). 